\title{
Hong-Ou-Mandel Interference with Imperfect Single Photon Sources
}

\author{
H. Ollivier, ${ }^{1}$ S. E. Thomas $\odot,{ }^{1,}$ ' S. C. Wein $\odot,{ }^{2}$ I. Maillette de Buy Wenniger $\odot,{ }^{1}$ N. Coste, ${ }^{1}$ J. C. Loredo $\odot,{ }^{1}$ N. Somaschi, ${ }^{3}$ \\ A. Harouri, ${ }^{1}$ A. Lemaitre $\odot,{ }^{1}$ I. Sagnes $\odot,{ }^{1}$ L. Lanco $\odot,{ }^{1,4}$ C. Simon $\odot,{ }^{2}$ C. Anton $\odot,{ }^{1}$ O. Krebs $\odot,{ }^{1}$ and P. Senellart ${ }^{1, \dagger}$ \\ ${ }^{1}$ Centre for Nanosciences and Nanotechnology, CNRS, Université Paris-Saclay, \\ UMR 9001, 10 Boulevard Thomas Gobert, 91120 Palaiseau, France \\ ${ }^{2}$ Institute for Quantum Science and Technology and Department of Physics and Astronomy, University of Calgary, \\ Calgary, Alberta, Canada T2N 1N4 \\ ${ }^{3}$ Quandela SAS, 10 Boulevard Thomas Gobert, 91120 Palaiseau, France \\ ${ }^{4}$ Université Paris Diderot-Paris 7, 75205 Paris CEDEX 13, France
}

(Received 4 May 2020; accepted 8 October 2020; published 12 February 2021)

\begin{abstract}
Hong-Ou-Mandel interference is a cornerstone of optical quantum technologies. We explore both theoretically and experimentally how unwanted multiphoton components of single-photon sources affect the interference visibility, and find that the overlap between the single photons and the noise photons significantly impacts the interference. We apply our approach to quantum dot single-photon sources to access the mean wave packet overlap of the single-photon component. This study provides a consistent platform with which to diagnose the limitations of current single-photon sources on the route towards the ideal device.
\end{abstract}

DOI: 10.1103/PhysRevLett.126.063602

Quantum interference of indistinguishable single photons is a critical element of quantum technologies. It allows the implementation of logical photon-photon gates for quantum computing [1,2] as well as the development of quantum repeaters for secure long distance communications $[3,4]$. The development of efficient sources of single and indistinguishable photons has become a challenge of the utmost importance in this regard, with two predominant, distinct approaches. The first one is based on nonlinear optical photon pair production [5,6], and multiplexing of heralded single photon sources is being explored to overcome an intrinsic inefficiency [7-10]. The other is based on single quantum emitters such as semiconductor quantum dots $[11,12]$ where ever-growing control of the solid-state emitter has enabled the combination of high efficiency and high indistinguishability [13-16].

The standard method to quantify the indistinguishability of single-photon wavepackets is to perform Hong-OuMandel (HOM) interference [17]. In perfect HOM interference, two indistinguishable single photons incident at each input of a 50:50 beam splitter will exit the beam splitter together, resulting in no two-photon coincidental detection events at both outputs. In practice, however, the two inputs only exhibit partial indistinguishability

Published by the American Physical Society under the terms of the Creative Commons Attribution 4.0 International license. Further distribution of this work must maintain attribution to the author(s) and the published article's title, journal citation, and DOI. described by a nonunity mean wave packet overlap $M_{\mathrm{s}}$ (also defined as the single-photon trace purity $[18,19]$ ). Partial indistinguishability of the input states leads to coincidental detection events at the outputs and reduces the HOM interference visibility. The interference visibility $V_{\text {HOM }}$ can therefore give direct access to the single-photon indistinguishability, $M_{\mathrm{s}}=V_{\mathrm{HOM}}[19]$.

For nonideal single-photon sources, for which the photonic wave packets present a residual multiphoton component, the HOM visibility remains the relevant quantity that determines the quality of the above-mentioned quantum operations. However, the visibility of HOM interference is reduced due to multiphoton contributions, even if $M_{\mathrm{s}}=1$, i.e., for an ideal single-photon indistinguishability. In most cases, the multiphoton component of the photonic wave packet, characterized by the second order intensity autocorrelation at zero time delay $g^{(2)}(0)$, depends on the system parameters in a manner that is completely independent of the single photon indistinguishability, and it is critical to have tools to access the latter in order to understand the physics at play and improve the performance of single photon sources (SPSs).

Here we explore both theoretically and experimentally HOM interference with imperfect SPSs. Previously, the impact of multiphoton contributions on HOM interference has been investigated in the limited case where the additional photons are in the same spectral and temporal mode as the predominant ones [20-22]. It has been shown that the visibility of HOM interference in this case is given by $V_{\text {HOM }}=M_{\text {tot }}-g^{(2)}(0)[19,23,24]$, where $M_{\text {tot }}$ is the mean wave packet overlap of the total input state, i.e., including 
the multiphoton component. Here we show that the properties of the additional or "noise" photons play a critical role in HOM interference, and that it is crucial to know the origin of the imperfections to be able to correctly extract the intrinsic single-photon indistinguishability $M_{\mathrm{s}}$.

We model an imperfect "single-photon" state $\left[g^{(2)}(0)>0\right]$ by mixing a true single photon $\left[g^{(2)}(0)=0\right]$ with separable noise at a beam splitter. We limit our analysis to small $g^{(2)}(0)$ values so that the noise field itself is well approximated by an optical field with at most one additional photon and a large vacuum contribution. This restriction to a weak, separable noise field is relevant in practice for many SPSs, as discussed later.

It can be shown (see Supplemental Material [25]), that for separable noise and a small resultant $g^{(2)}$ (typically $\left.g^{(2)}<0.3\right)$, the visibility of HOM interference at a 50:50 beam splitter is given by

$$
V_{\mathrm{HOM}}=M_{\mathrm{s}}-\left(\frac{1+M_{\mathrm{s}}}{1+M_{\mathrm{sn}}}\right) g^{(2)},
$$

where $M_{\mathrm{sn}}$ is the mean wave packet overlap between the single photon and an additional noise photon satisfying $0 \leq M_{\text {sn }} \leq M_{\mathrm{s}}$. We have defined $g^{(2)} \equiv g^{(2)}(0)$ for simplicity. The more general case where the HOM beam splitter has an intensity reflectivity $R$ and transmission $T$ is given in the Supplemental Material [25].

It is instructive to consider the two limiting cases of Eq. (1). If the additional photons are identical to the single photons, i.e., $M_{\mathrm{sn}}=M_{\mathrm{s}}$, then Eq. (1) reduces to the simple case that $V_{\mathrm{HOM}}=M_{\mathrm{s}}-g^{(2)}$, showing that the total and single photon mean wave packet overlaps coincide, $M_{\mathrm{s}}=M_{\text {tot }}$. Alternatively, if the noise has no overlap with the single photons and $M_{\mathrm{sn}}=0$, then the visibility is further reduced and given by $V_{\mathrm{HOM}}=M_{\mathrm{s}}-\left(1+M_{\mathrm{s}}\right) g^{(2)}$. The degree to which HOM interference is affected by a nonzero $g^{(2)}$ is therefore dependent on the origin of the additional photons.

We experimentally test this model by emulating imperfect SPSs. We prepare a train of near-optimal single photons and mix them with additional photons to controllably increase $g^{(2)}$ and measure the impact on the HOM interference. We experimentally emulate the two limiting cases, $M_{\mathrm{sn}}=0$ and $M_{\mathrm{sn}}=M_{\mathrm{s}}$, and in each case we measure the $g^{(2)}$ and HOM interference visibility of the resultant wave packet in an unbalanced Mach-Zehnder interferometer (see Supplemental Material [25] for further details).

We use a state-of-the-art SPS based on a quantum dot (QD) deterministically embedded in an electrically contacted micropillar cavity [13]. The QD acts as an artificial atom which we coherently control via resonant excitation to generate single photons with high single photon purity, $g^{(2)}<0.05$, and high indistinguishability, $M_{\text {tot }}>0.9$. The single photons are separated from the excitation laser using a cross-polarization setup, as shown in Fig. 1(a).

The experimental setups that enable a controlled increase of the multiphoton probability are shown in Figs. 1(b) and 1(c) for the two limiting cases. First, to add fully distinguishable photons, we mix the single photons from the quantum dot based single photon source (QDSPS) with attenuated laser pulses at a different wavelength (see Supplemental Material [25] for more details). We adjust the power of the laser beam to alter the magnitude of the two-photon component, and measure the HOM visibility as a function of $g^{(2)}$ of this effective source as shown in Fig. 1(d). Since the spectral overlap between the QDSPS photons and the additional laser photons is zero $\left(M_{\mathrm{sn}}=0\right)$ our model predicts that $V_{\mathrm{HOM}}=M_{\mathrm{s}}-\left(1+M_{\mathrm{s}}\right) g^{(2)}$. The line in Fig. 1(d) shows that this model fits the data very well with $M_{\mathrm{s}}=0.94 \pm 0.02$.

To create a wave packet where the additional photons are identical to the predominant single photon component, we build another effective source where we add a small fraction

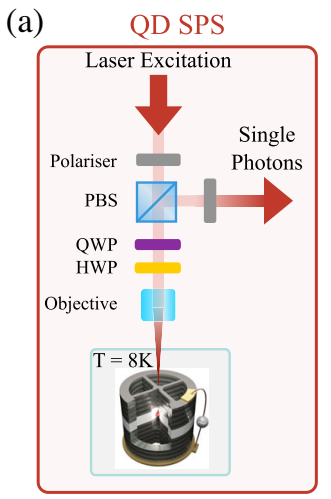

(b)

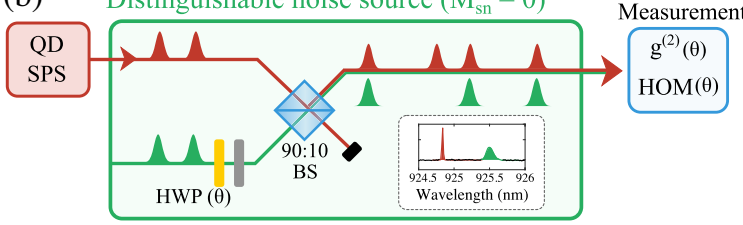

(c)

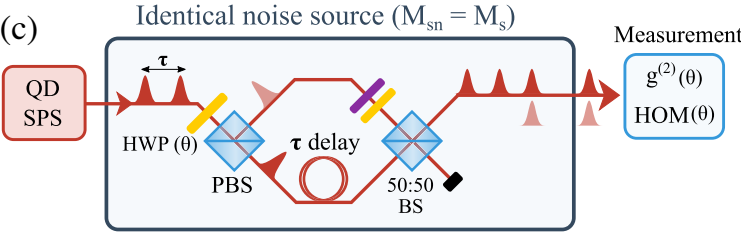

(d)

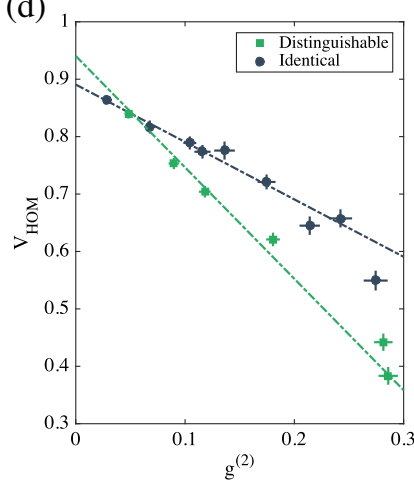

FIG. 1. (a) Schematic for the experimental setup of a quantum dot single photon source (QDSPS). For more details see the Supplemental Material [25]. (b),(c) Experimental setup used to emulate an imperfect single photon source with (b) distinguishable $\left(M_{\mathrm{sn}}=0\right)$ and (c) identical noise $M_{\mathrm{sn}}=M_{\mathrm{s}}$. The inset in (b) shows the spectrum of the spectrally distinct noise photons and QD photons. (d) Visibility of HOM interference, $V_{\mathrm{HOM}}$ measured as a function of $g^{(2)}$ for distinguishable (green squares) and identical (black circles) noise sources. The lines are the predictions from the theoretical model. 
of photons from the same QDSPS generated at a later time. This is obtained by performing an unbalanced quantum interference between two photon pulses produced by the QDSPS with delay $\tau$. The first half wave plate (HWP) and polarizing beam splitter (PBS) in Fig. 1(c) allow us to tune the relative intensity of the predominant single photon pulse and the additional photons. When two subsequently emitted photons arrive simultaneously at the second beam splitter they will undergo HOM interference and exit the beam splitter in the same output port. Therefore, the output of the second BS has a higher $g^{(2)}$, since there is a small probability that some of the output pulses now contain two identical photons. By adjusting the splitting ratio at the first beam splitter, the $g^{(2)}$ of the output state can be controlled. Figure 1(d) presents the HOM visibility as we increase the $g^{(2)}$ via addition of identical photons, where a clear difference is observed compared to the previous limiting case. For $M_{\mathrm{sn}}=M_{\mathrm{s}}$, the model predicts $V_{\mathrm{HOM}}=M_{\mathrm{s}}-g^{(2)}$, a linear dependence with slope of -1 . The line in Fig. 1(d) again demonstrates that the model gives a very good fit to the data, with an extracted $M_{\mathrm{s}}=0.89 \pm 0.01$.

We note that the extracted values of $M_{\mathrm{s}}$ for these two cases represent the upper and lower bound of the intrinsic single photon indistinguishability of the QDSPS used in these measurements. If the nonzero $g^{(2)}$ of the QDSPS was due to distinguishable noise then we could deduce that $M_{\mathrm{s}}=0.94 \pm 0.02$. Similarly if the noise was identical then the QDSPS has a single photon indistinguishability of $M_{\mathrm{s}}=0.89 \pm 0.01$. This demonstrates that it is necessary to know the origin of the unwanted photon emission in order to be able to extrapolate the data back to $g^{(2)}=0$. To the extent of our knowledge, this has so far only been done in the indistinguishable case, independent of the physical phenomena of the multi-photon components. In the following, we discuss how to properly estimate the single photon indistinguishability for QD based SPSs.

There are two distinct categories of QDSPS, depending on the charge state of the quantum dot: neutral excitons and charged excitons (hereafter referred to as exciton and trion states, respectively). The optical selection rules and photon emission processes differ significantly between the excitons and trions [26], leading to a different origin of the multiphoton component.

For a trion based source, the optical selection rules correspond to a four level system with four possible linearly polarized transitions [27]. In a cross-polarized setup, this system behaves like an effective two-level system, and the single photon emission shows a rapid rise time and monoexponential decay as shown in Fig. 2(a). Since the single photon emission process is fast, it can occur during the laser pulse so that there is a probability that the quantum dot returns to the ground state before the end of the laser pulse and gets excited again, leading to the emission of a second photon [28]. Therefore, the nonzero $g^{(2)}$ for a trion based source is predominantly due to reexcitation.
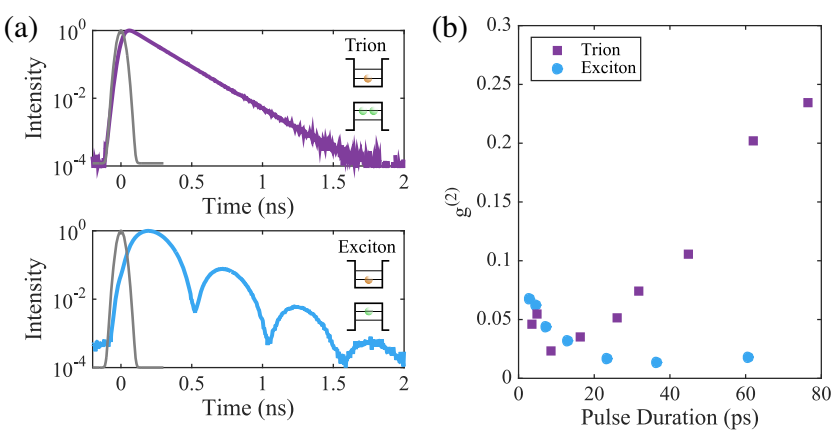

FIG. 2. (a) Time traces of the single photon wave packet emitted by trion (upper) and exciton (lower) sources. The excitation laser pulse is shown in gray. (b) Measured $g^{(2)}$ for trion (purple squares) and exciton (blue circles) sources as a function of the excitation pulse duration at $\pi$ pulse. The error bars are within the size of the plotted points.

For an exciton, the system is described by a three level system where the excitation pulse creates a superposition of the two excitonic linear dipoles with an energy difference given by the fine-structure splitting [29]. This results in a time-dependent phase between the two exciton eigenstates, so that the single-photon emission in cross polarization beats with a period determined by the fine structure splitting [26,30], as shown in Fig. 2(a). These optical selection rules imply that the single photon emission in cross polarization is delayed with respect to the excitation pulse, and this delay means that the probability of collecting two photons via reexcitation is very small.

To illustrate this, we measure the $g^{(2)}$ at maximum emitted brightness for two QD sources, one exciton and one trion, while increasing the temporal length of the excitation pulse [Fig. 2(b)]. For the exciton source, the single photon purity remains very high for pulse durations up to $80 \mathrm{ps}$, whereas the single photon purity rapidly degrades for longer pulses for the trion based source due to an increased probability of reexcitation. We notice that for both excitons and trions, the $g^{(2)}$ is higher for very short pulses. This is because the power required to reach maximum emitted brightness ( $\pi$ pulse) increases as the pulse duration decreases [31]. For a fixed pulse area, the Rabi frequency of a pulse of duration $\tau$ is proportional to $1 / \tau$, corresponding to a pulse power proportional to $1 / \tau^{2}$ and a total energy per pulse $(\tau \times P)$ proportional to $1 / \tau$. This implies that in the very short pulse regime $(<10 \mathrm{ps})$, more excitation power is required to reach $\pi$ pulse and the $g^{(2)}$ is limited by imperfect suppression of the excitation laser. This remains the dominant source of an imperfect $g^{(2)}$ for exciton sources up to a pulse duration of $80 \mathrm{ps,} \mathrm{whereas}$ trion sources are limited by reexcitation for pulses longer than 15 ps.

To correctly extract the single photon indistinguishability for each type of QDSPS it is critical to account for these different origins of the multiphoton component. To do so, 
we experimentally increase the multiphoton component by adjusting the main parameter that is responsible for multiphoton emission in each type of source, and then measure the impact this has on the visibility of HOM interference. By deliberately introducing noise with the same origin as that which limits the $g^{(2)}$ for each type of source, we ensure that we can extrapolate the relationship between $g^{(2)}$ and $V_{\text {HOM }}$ to values below the lowest $g^{(2)}$ that we can measure. Specifically, we increase the probability of reexcitation for a trion source and the amount of laser photons for the exciton source.

For the exciton based source, we have two different methods to add laser photons to the single photon emission from the QDSPS. First, we can decrease the pulse duration which, as described above, means that we require more power to reach $\pi$ pulse, and therefore there is a higher probability of detecting a laser photon. Second, we can rotate the quarter-wave plate (QWP) of the excitation pulse [see Fig. 1(a)], so that the excitation polarization is no longer aligned along one of the polarization axes of the cavity. The light will experience polarization rotation due to the birefringence of the cavity, and some fraction of the excitation pulse will now be collected in the orthogonal polarization with the single photons. By adjusting the QWP we can decrease the polarization extinction of the laser and add more laser photons. We increase the $g^{(2)}$ of the effective source according to each of these methods, and measure the corresponding impact on HOM interference as shown in Fig. 3(a). We fit Eq. (1) to the data (since the added noise from the laser is separable), and we extract $M_{\mathrm{S}}=0.94 \pm$ $0.01\left(M_{\mathrm{s}}=0.91 \pm 0.01\right)$ and $M_{\mathrm{sn}}=-0.02 \pm 0.09\left(M_{\mathrm{sn}}=\right.$ $0.03 \pm 0.06)$ for the case where we change the pulse duration (polarization extinction). In both cases, the extracted value of $M_{\mathrm{sn}}$ is equal to zero within error. From Fig. 2(b), we can indeed see that there is very little overlap between the laser photons and the single photons emitted by the quantum dot, an observation compatible with $M_{\mathrm{sn}}=0$. We note that the two datasets were
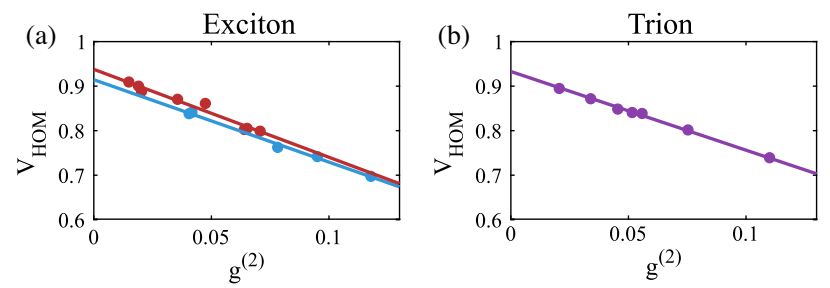

FIG. 3. (a) Measured HOM visibility as a function of the $g^{(2)}$ for an exciton source. The $g^{(2)}$ is increased by either decreasing the pulse duration of the excitation pulse (red), or decreasing the suppression of the excitation laser (blue). The two methods are performed on different exciton sources. (b) Measured HOM visibility as a function of the $g^{(2)}$ for a trion source as the pulse duration is increased. In both plots, the solid line gives the theoretical prediction for these data. The error bars are within the size of the plotted points. performed on different excitons which explains the slight difference in $M_{\mathrm{s}}$ of the two devices.

For the trion based source, since the imperfect $g^{(2)}$ arises from reexcitation, the assumption of separable noise does not hold because the emission of the first and second photon are time correlated. However, the extra photon must be emitted during the laser pulse for re-excitation to occur [28], whereas the main single photon emission typically takes place after the laser pulse with the trion radiative decay time of approximately $170 \mathrm{ps}$. As a result, the noise photon, while emitted by the QD, is temporally distinguishable from the predominant single-photon emission. This noise can thus be considered mostly separable and distinguishable. The validity of this analysis is verified by increasing the pulse duration to increase the probability of reexcitation. The $g^{(2)}$ and HOM visibility are measured for different pulse durations from 15 to $50 \mathrm{ps}$ at $\pi$ pulse, and the results shown in Fig. 3(b). We fit our observations with Eq. (1) and find $M_{\mathrm{s}}=0.93 \pm 0.01$ and $M_{\mathrm{sn}}=0.09 \pm 0.02$, indicating that the overlap between the noise photons and the single photons from the QDSPS is indeed very low. In fact, if we fit the data using the distinguishable noise model $\left(M_{\mathrm{sn}}=0\right)$ we extract a single parameter $M_{\mathrm{s}}=0.94 \pm 0.01$, which is in good agreement with the value extracted using Eq. (1).

For both the exciton and trion based sources we extract a single-photon indistinguishability, $M_{\mathrm{s}}$, of around 0.93 . The residual distinguishability is due to phonon-assisted emission into the phonon sideband, as well as pure dephasing of the zero phonon line. Both of these effects are reduced by cavity quantum electrodynamics effects, and very high values of indistinguishability have been measured in high $Q$-factor cavities [32]. However, the current implementation is in a lower $Q$-factor cavity, and some phononinduced decoherence remains.

To summarize, we find that, despite their different physical origins, the multiphoton component of both exciton and trion based QDSPSs can be treated as separable distinguishable noise. In the limit of low $g^{(2)}$, the single photon indistinguishability can thus be obtained using

$$
M_{\mathrm{s}}=\frac{V_{\mathrm{HOM}}+g^{(2)}}{1-g^{(2)}} .
$$

This correction factor allows one to extract the intrinsic single photon indistinguishability $M_{\mathrm{s}}$, given a measurement of $g^{(2)}$ and $V_{\mathrm{HOM}}$.

We note that, among the large number of works dedicated to QDSPSs, the correction factor that has been used to account for an imperfect $g^{(2)}$ differs significantly, with some using $M=V_{\mathrm{HOM}}+g^{(2)} \quad[32,33]$, others using $M=$ $V_{\mathrm{HOM}}+2 g^{(2)}[13,16]$, and some using $M=V_{\mathrm{HOM}} \times(1+$ $\left.2 g^{(2)}\right)$ [34], with none of them discussing the nature of the noise. For the cases where the lower bound was taken, our study shows that for these QDSPS the actual 
indistinguishability is larger than previously claimed. The correction factor derived here has been applied to a recent benchmarking of multiple QDSPSs based on both charged and neutral excitons [26]. It was found that, despite the $g^{(2)}$ of the different sources varying between $2 \%$ and $7 \%$, the extracted single-photon indistinguishability was consistently between $92 \%$ and $95 \%$ for 12 of the sources.

We finally note that our approach applies to many SPSs, including all SPSs based on single quantum emitters, whether the residual $g^{(2)}$ arises from imperfect laser filtering or re-excitation. It is also applicable to SPSs based on nonlinear optical frequency conversion where the nonzero $g^{(2)}$ is predominantly due to multiple photon-pair generation. For single-mode sources, the two-photon component is described by a two-photon Fock state with indistinguishable photons in a single, well-defined mode. This can be reproduced by the beam splitter model presented here via photon bunching, and hence the separable noise model with indistinguishable noise photons applies. The case of multi photon-pair generation in multimode sources, however, would require further investigations.

In conclusion, we have theoretically and experimentally revisited the emblematic Hong-Ou-Mandel interference. This experiment is commonly implemented to test the indistinguishability of single particles including single photons, single plasmons, single electrons, or single atoms [35-37]. We believe that the new insight brought by our study will benefit these fundamental studies as well as the development of single photon sources. Here we have showed how to extract the single-photon indistinguishability $M_{\mathrm{s}}$, which gives the upper bound to the indistinguishability that could be achieved with an ideal experimental setup and fundamentally quantifies how temporally coherent the source itself is. While this does not circumvent the fact that it is the overall wave packet indistinguishability that is crucial for quantum technologies, this deepened understanding of the Hong-Ou-Mandel experiment enables us to carefully account for the impact of multiphoton events on the HOM visibility which is essential to properly identify the remaining limitations in the journey towards an ideal SPS.

This work was partially supported by the ERC PoC PhoW, the French Agence Nationale pour la Recherche (grant ANR QuDICE), the IAD-ANR support ASTRID program Projet No. ANR-18-ASTR-0024 LIGHT, the QuantERA ERA-NET Cofund in Quantum Technologies, project HIPHOP, the French RENATECH network, a public grant overseen by the French National Research Agency (ANR) as part of the "Investissements dAvenir" programme (Labex NanoSaclay, reference: ANR-10-LABX-0035). J. C. L. and C. A. acknowledge support from Marie SklodowskaCurie Individual Fellowships SMUPHOS and SQUAPH, respectively. H. O. acknowledges support from the Paris Ile-de-France Rgion in the framework of DIM SIRTEQ. S. C.W. and C.S. acknowledge support from NSERC (the Natural Sciences and Engineering Research Council), AITF (Alberta Innovates Technology Futures), and the SPIE Education Scholarships program. P. S. acknowledges the support from the iXcore Foundation for research.

H. O. and S. E. T. contributed equally to this work.

*sarah.thomas@c2n.upsaclay.fr pascale.senellart-mardon@c2n.upsaclay.fr

[1] E. Knill, R. Laflamme, and G. J. Milburn, Nature (London) 409, 46 (2001).

[2] J. L. O'Brien, Science 318, 1567 (2007).

[3] L.-M. Duan, M. D. Lukin, J. I. Cirac, and P. Zoller, Nature (London) 414, 413 (2001).

[4] N. Sangouard, C. Simon, H. de Riedmatten, and N. Gisin, Rev. Mod. Phys. 83, 33 (2011).

[5] P. G. Kwiat, E. Waks, A. G. White, I. Appelbaum, and P. H. Eberhard, Phys. Rev. A 60, R773 (1999).

[6] H.-S. Zhong et al., Phys. Rev. Lett. 121, 250505 (2018).

[7] F. Kaneda and P. G. Kwiat, Sci. Adv. 5, eaaw8586 (2019).

[8] C. Joshi, A. Farsi, S. Clemmen, S. Ramelow, and A. L. Gaeta, Nat. Commun. 9, 847 (2018).

[9] C. Xiong, X. Zhang, Z. Liu, M. J. Collins, A. Mahendra, L. G. Helt, M. J. Steel, D.-Y. Choi, C. J. Chae, P. H. W. Leong, and B. J. Eggleton, Nat. Commun. 7, 10853 (2016).

[10] R. J. A. Francis-Jones, R. A. Hoggarth, and P. J. Mosley, Optica 3, 1270 (2016).

[11] I. Aharonovich, D. Englund, and M. Toth, Nat. Photonics 10, 631 (2016).

[12] P. Senellart, G. Solomon, and A. White, Nat. Nanotechnol. 12, 1026 (2017).

[13] N. Somaschi, V. Giesz, L. De Santis, J. C. Loredo, M. P. Almeida, G. Hornecker, S. L. Portalupi, T. Grange, C. Antón, J. Demory, C. Gómez, I. Sagnes, N. D. Lanzillotti-Kimura, A. Lemaítre, A. Auffeves, A. G. White, L. Lanco, and P. Senellart, Nat. Photonics 10, 340 (2016).

[14] H. Wang, Z.-C. Duan, Y.-H. Li, S. Chen, J.-P. Li, Y.-M. He, M.-C. Chen, Y. He, X. Ding, C.-Z. Peng, C. Schneider, M. Kamp, S. Höfling, C.-Y. Lu, and J.-W. Pan, Phys. Rev. Lett. 116, 213601 (2016).

[15] Y.-M. He, H. Wang, C. Wang, M. C. Chen, X. Ding, J. Qin, Z. C. Duan, S. Chen, J. P. Li, R.-Z. Liu, C. Schneider, M. Atatüre, S. Höfling, C.-Y. Lu, and J.-W. Pan, Nat. Phys. 15, 941 (2019).

[16] H. Wang et al., Nat. Photonics 13, 770 (2019).

[17] C. K. Hong, Z. Y. Ou, and L. Mandel, Phys. Rev. Lett. 59, 2044 (1987).

[18] K. A. Fischer, R. Trivedi, and D. Lukin, Phys. Rev. A 98, 023853 (2018).

[19] R. Trivedi, K. A. Fischer, J. Vukovi, and K. Mller, Adv. Quantum Technol. 3, 1900007 (2020).

[20] A. J. Bennett, R. B. Patel, C. A. Nicoll, D. A. Ritchie, and A. J. Shields, Nat. Phys. 5, 715 (2009).

[21] S. V. Polyakov, A. Muller, E. B. Flagg, A. Ling, N. Borjemscaia, E. Van Keuren, A. Migdall, and G.S. Solomon, Phys. Rev. Lett. 107, 157402 (2011).

[22] T. Huber, M. Prilmller, M. Sehner, G. S. Solomon, A. Predojević, and G. Weihs, Quantum Sci. Technol. 2, 034016 (2017). 
[23] A. Kiraz, M. Atatüre, and A. Imamoğlu, Phys. Rev. A 69, 032305 (2004).

[24] A. B. U'Ren, C. Silberhorn, J. L. Ball, K. Banaszek, and I. A. Walmsley, Phys. Rev. A 72, 021802(R) (2005).

[25] See Supplemental Material at http://link.aps.org/ supplemental/10.1103/PhysRevLett.126.063602 for a theoretical derivation of the relationship between Hong-OuMandel interference visibility and single-photon purity in a separable noise model, as well as further experimental details.

[26] H. Ollivier, I. Maillette de Buy Wenniger, S. Thomas, S. C. Wein, A. Harouri, G. Coppola, P. Hilaire, C. Millet, A. Lemaitre, I. Sagnes, O. Krebs, L. Lanco, J. C. Loredo, C. Anton, N. Somaschi, and P. Senellart, ACS Photonics 7, 1050 (2020).

[27] X. Xu, Y. Wu, B. Sun, Q. Huang, J. Cheng, D. G. Steel, A. S. Bracker, D. Gammon, C. Emary, and L. J. Sham, Phys. Rev. Lett. 99, 097401 (2007).

[28] K. A. Fischer, L. Hanschke, M. Kremser, J. J. Finley, K. Mller, and J. Vučković, Quantum Sci. Technol. 3, 014006 (2017).

[29] M. Bayer, G. Ortner, O. Stern, A. Kuther, A. A. Gorbunov, A. Forchel, P. Hawrylak, S. Fafard, K. Hinzer, T. L. Reinecke, S. N. Walck, J.P. Reithmaier, F. Klopf, and F. Schäfer, Phys. Rev. B 65, 195315 (2002).

[30] A. S. Lenihan, M. V. Gurudev Dutt, D. G. Steel, S. Ghosh, and P. K. Bhattacharya, Phys. Rev. Lett. 88, 223601 (2002).
[31] V. Giesz, N. Somaschi, G. Hornecker, T. Grange, B. Reznychenko, L. De Santis, J. Demory, C. Gomez, I. Sagnes, A. Lemaitre, O. Krebs, N. D. Lanzillotti-Kimura, L. Lanco, A. Auffeves, and P. Senellart, Nat. Commun. 7, 11986 (2016).

[32] T. Grange, N. Somaschi, C. Antón, L. De Santis, G. Coppola, V. Giesz, A. Lemaître, I. Sagnes, A. Auffèves, and P. Senellart, Phys. Rev. Lett. 118, 253602 (2017).

[33] G. Kirsanske, H. Thyrrestrup, R. S. Daveau, C. L. Dreeßen, T. Pregnolato, L. Midolo, P. Tighineanu, A. Javadi, S. Stobbe, R. Schott, A. Ludwig, A. D. Wieck, S. I. Park, J. D. Song, A. V. Kuhlmann, I. Söllner, M. C. Löbl, R. J. Warburton, and P. Lodahl, Phys. Rev. B 96, 165306 (2017).

[34] N. Tomm, J. Javadi, N. O. Antoniadis, D. Najer, M. C. Lobl, A. R. Korsch, R. Schott, S. R. Valentin, A. D. Wieck, A. Ludwig, and R. J. Warburton, arXiv:2007.12654.

[35] G. Di Martino, Y. Sonnefraud, M. S. Tame, S. Kéna-Cohen, F. Dieleman, S. K. Özdemir, M. S. Kim, and S. A. Maier, Phys. Rev. Applied 1, 034004 (2014).

[36] E. Bocquillon, V. Freulon, J.-M. Berroir, P. Degiovanni, B. Plaçais, A. Cavanna, Y. Jin, and G. Fève, Science 339, 1054 (2013).

[37] R. Lopes, A. Imanaliev, A. Aspect, M. Cheneau, D. Boiron, and C. I. Westbrook, Nature (London) 520, 66 (2015). 